\title{
Universal Service Obligations in Developing Countries
}

\author{
Antonio Estache* Jean-Jacques Laffont ${ }^{\dagger} \quad$ Xinzhu Zhang ${ }^{\ddagger}$
}

August, 2004

\begin{abstract}
This paper develops a model to analyze the impacts of asymmetric information on optimal universal service policy in the public utilities of developing countries. Optimal universal service policy is implemented using two regulatory instruments: pricing and network investment. Under discriminatory pricing asymmetric information leads to a higher price and smaller network in the rural area than under full information. Under uniform pricing the price is also lower but the network is even smaller. In addition, under both pricing regimes not only the firm but also taxpayers have incentives to collude with the regulator.

Keywords: Universal service obligations; Asymmetric information; Collusion

JEL Classification: L43; D82; O12
\end{abstract}

\section{Introduction}

Universal service obligations have played a prominent role in policy debates on public utilities in developed economies. ${ }^{1}$ In the United States, for instance, a large part of the 1996 Telecommunications Act is devoted to reforming universal service obligations, and the Federal Communications Commission has made enormous efforts to implement these obligations. Governments in developing

\footnotetext{
${ }^{*}$ Corresponding author. World Bank, Washington, D.C. 20433 and ECARES, Universite Libre de Bruxelles. Tel.: +1-202-458-1442; fax: +1-202-522-2961. Email Address: aestache@worldbank.org (A. Estache).

${ }^{\dagger}$ IDEI and Université des Sciences Sociales, Place Anatole, 31042 Toulouse Cedex, France. Professor Laffont passed away on May 1, 2004.

${ }^{\ddagger}$ Research Center for Regulation and Competition, Chinese Academy of Social Sciences, Beijing 100732, P. R. China.

${ }^{1}$ For recent overview of the economics of USO, see Cremer and et al. (2001).
} 
countries have also viewed these obligations as an important social commitment and implemented universal service policies - to varying degrees - as part of regulatory reforms (see Clarke and Wallsten (2002) for an overview). For developing countries universal service obligations are broadly viewed by politicians and policy makers as a necessary component of equitable development strategies - redistribution toward the poor and the underdeveloped regions as part of USOs.

Still, universal service obligations have created considerable controversies in reform processes and raised many practical and theoretical issues - two of which are especially relevant to developing countries. ${ }^{2}$ The first is how best to promote network expansion. Unlike in industrial countries, where penetration of basic infrastructure services is no longer an issue, ${ }^{3}$ in developing countries many people still lack these services, particularly in rural areas. Thus network expansion remains an essential dimension of universal service policies in developing countries.

The other important issue in developing countries is the design of pricing policy instruments to support universal service obligations. Because the necessary fiscal instruments are often not available, policy makers have traditionally tended to rely mostly on simple pricing policies to achieve both allocative efficiency and redistribution goals. USOs have indeed often been financed by cross-subsidies across clients of the monopoly responsible for service delivery. But this historical approach needs to be reassessed once competition is introduced in a sector and regulatory decisions associated with the specific economic characteristics of the sector and the service users need to be designed. This is a particularly difficult challenge in developing countries which generally have limited experience in regulating infrastructure in a competitive environment and where regulatory capture risks may be associated with institutional limitations.

\footnotetext{
${ }^{2}$ There is very little academic literature on universal services obligations in developing countries. For an overview of the developing countries experience in adopting USO, see Chisari et al. (2003), Clarke-Wallsten (2002), Estache et al. (2003), Laffont and N'Gbo (2000) or Gasmi et al. (1999).

${ }^{3}$ People might object that what is "basic" get redefined. So "Internet access has now become a subject of debate as many now still don't have access to broadband access in developed countries.
} 
The main contribution of this paper is to use incentive theory to analyze these specific policy issues in developing countries. It provides a first step toward understanding the theoretical foundations of universal service policies from a normative point of view, and the associated features of political economy in developing countries. To do so, the paper develops a simple model based on asymmetric information, in which a developing country government lacks information about a monopolistic firm's marginal cost of providing an infrastructure service in a rural area. The model is used to analyze the impacts of asymmetric information and the threat of regulatory capture on optimal universal service policy in the public utilities of developing countries. Here optimal universal service policy is implemented using two regulatory instruments: pricing and network investment. The analysis is conducted under both discriminatory pricing (between urban and rural areas) and uniform pricing. The results show that under both pricing regimes, asymmetric information results in a higher price and smaller network in the rural area than does complete information. More importantly, although uniform pricing leads to a lower price in the rural area, the network size is smaller than under discriminatory pricing.

When considering the possibilities of collusion between various actors, the analysis here relies on the hard information structure used in Tirole (1986) and Laffont and Tirole (1993). An important feature of our model is that multiple interest groups have incentives to collude with the regulator. The firm, which obtains an information rent if the regulator does not reveal its private information, has incentives to collude with the regulator if it is relying on a low-cost technology. At the same time, taxpayers may benefit from information hiding by the regulator (by paying less taxes) if the firm uses a high-cost technology. ${ }^{4}$ Although collusion between the regulator and taxpayers may be difficult- since taxpayers are often not well organized - our analysis points out the stakes that taxpayers may have in a specific universal service policy. Preventing collusion between the regulator and the firm requires increasing the price and reducing the size of the network for the rural area. But prevention of collusion between

\footnotetext{
${ }^{4}$ In developed countries and some developing economies, USO cost is usually born by users in other regions or richer users rather than by taxpayers. So the relevant collusion would be with a roundtable of metropolitan areas rather than with taxpayers.
} 
the regulator and taxpayers needs opposite regulatory responses. Therefore, the existence of taxpayers as an interest group hardens the coalition incentive constraint for the regulator and the firm.

The rest of the paper is organized as follows: Section 2 establishes the basic setting for the analysis and the resulting model. After that optimal regulation of pricing is considered, with analysis of discriminatory pricing in Section 3 and uniform pricing in Section 4. We analyze the impacts of the threat of collusion in section 5. Section 6 then concludes.

\section{The Basic Setting}

Consider a simple principal-agent relationship between a benevolent government and a monopolistic firm that is regulated when providing, for instance, telecommunications services in a specific territory. The firm's territory is divided into two areas: a low-cost urban one and a high-cost rural one. Let $\alpha_{1}$ be the share of the population in the urban area and $\alpha_{2}$ the share in the rural area, with $\alpha_{1}+\alpha_{2}=1$.

Assume that the marginal cost of providing services in the urban area is $c_{1}$, which is common knowledge. One can think of this assumption as a reduced form of competition in the urban sector. But the government has asymmetric information about $c_{2}$, the marginal cost of providing services in the rural area. For simplicity assume that there are only two possible cost levels or two typesthat is, $c_{2} \in\left\{\underline{c}_{2}, \bar{c}_{2}\right\}$ and $c_{2}>c_{1}$, with probability distribution $\operatorname{Pr}\left(c_{2}=\underline{c}_{2}\right)=\nu$ and $\operatorname{Pr}\left(c_{2}=\bar{c}_{2}\right)=1-\nu$. Let $\Delta c_{2} \equiv \bar{c}_{2}-\underline{c}_{2}>0$.

Assume also that full service coverage has been achieved in the urban area but that the rural area is only partially connected. Denote $\mu<1$ the proportion of households in the rural area which are connected. Because some people in the rural area are not connected, the government imposes universal service obligations on the monopolistic firm in the form of specified pricing and network expansion in the rural area. Assume that the cost of network investment $C(\mu)$ is a convex function satisfying $C^{\prime}(\cdot)>0$ and $C^{\prime \prime}(\cdot)>0$. 
Let $S(q)$ be the gross social surplus for subscribers, derived from consumption of the service in both low-cost and high-cost areas, and $P(q)$ the corresponding inverse demand function. Assume that $\left|S^{\prime \prime}\right|$ is large enough throughout the paper so that the optimization programs are concave. To simplify our analysis, we do not consider consumers' connection decisions, which means that consumers derive the entire surplus from consumption of services and all capacities are used immediately, because we focus on linear pricing which is one way of getting users to enjoy a rent from getting connected; alternatively, users could be heterogeneous and non linear pricing could be used. We also assume for simplicity that consumers have a demand with constant absolute elasticity $\eta>1$.

The timing of events is as follows. After the firm learns its private information about $c_{2}$, the government determines regulatory policies for services prices and network sizes in both urban and rural areas. The firm then invests to expand the rural network, and consumers make decisions about consumption.

\section{Optimal Regulation Under Price Discrimina- tion}

This section considers the case where price discrimination is allowed, so that the regulator sets different prices $\left(p_{1}\right.$ and $\left.p_{2}\right)$ for each area and consumers consume $q_{1}$ and $q_{2}$ accordingly. In that case the firm's utility function is:

$$
U=t+\alpha_{1} P\left(q_{1}\right) q_{1}+\alpha_{2} \mu P\left(q_{2}\right) q_{2}-\left[\alpha_{1} c_{1} q\left(p_{1}\right)+\alpha_{2} \mu c_{2} q\left(p_{2}\right)\right]-C(\mu),
$$

where $t$ is the monetary transfer obtained from the government since we do not constrain the firm to balance its budget. ${ }^{5}$

We assume that the benevolent regulator has a utilitarian social welfare

\footnotetext{
${ }^{5}$ Alternatively, the firm could have fixed costs and face a budget constraint. Then nothing changes except: (1) that $\lambda$ is the shadow cost of the budget constraint; (2) collusion is then with users in the urban area rather than with taxpayers, and (3) things will definitely be more complicated.
} 
function. Then,

$$
W=\alpha_{1}\left[S\left(q\left(p_{1}\right)\right)-p_{1} q\left(p_{1}\right)\right]+\alpha_{2} \mu\left[S\left(q\left(p_{2}\right)\right)-p_{2} q\left(p_{2}\right)\right]-(1+\lambda) t+U,
$$

where $\lambda>0$ is the shadow cost of public funds, which is exogenous. Substituting from (1) the transfer $t$, we can rewrite the objective function as:

$$
\begin{aligned}
W= & \alpha_{1}\left[S\left(q\left(p_{1}\right)\right)+\lambda p_{1} q\left(p_{1}\right)\right]+\alpha_{2} \mu\left[S\left(q\left(p_{2}\right)\right)+\lambda p_{2} q\left(p_{2}\right)\right] \\
& -(1+\lambda)\left[\alpha_{1} c_{1} q\left(p_{1}\right)+\alpha_{2} \mu c_{2} q\left(p_{2}\right)+C(\mu)\right]-\lambda U .
\end{aligned}
$$

For expositional simplicity the following notation is introduced:

$$
\bar{p}_{i}, \overline{q_{i}} \text { and } \underline{p}_{i}, \underline{q}_{i}
$$

are the price and quantity when $c_{2}$ is $\bar{c}_{2}$ and $\underline{c}_{2}$, respectively; and similarly

$$
\begin{array}{ll}
\bar{t}=t\left(\bar{c}_{2}\right) & \underline{t}=t\left(\underline{c}_{2}\right) \\
\bar{\mu}=\mu\left(\bar{c}_{2}\right) & \underline{\mu}=\mu\left(\underline{c}_{2}\right) .
\end{array}
$$

Before proceeding further, we derive the benchmark result under full information. The following participation constraints have to be satisfied to induce voluntary participation for the high-cost (or bad-type) firm:

$$
\bar{t}+\alpha_{1} \bar{p}_{1} \bar{q}_{1}+\alpha_{2} \overline{\mu p}_{2} \bar{q}_{2}-\left(\alpha_{1} c_{1} \bar{q}_{1}+\alpha_{2} \overline{\mu c}_{2} \bar{q}_{2}\right)-C(\bar{\mu}) \geq 0
$$

and for the low-cost (or good-type) firm:

$$
\underline{t}+\alpha_{1} \underline{p}_{1} \underline{q}_{1}+\alpha_{2} \underline{\mu p}_{2} \underline{q}_{2}-\left(\alpha_{1} c_{1} \underline{q}_{1}+\alpha_{2} \underline{\mu}_{2} \underline{q}_{2}\right)-C(\underline{\mu}) \geq 0 .
$$

So, the government's optimal regulatory policy under complete information can be obtained by solving the following program:

$$
\begin{array}{cl}
\operatorname{Max}_{\left\{\underline{q}_{1}, \underline{q}_{2}, \underline{\mu}, \underline{U}, \bar{q}_{1}, \bar{q}_{2}, \bar{\mu}, \bar{U}\right\}} & \nu\left\{\alpha_{1}\left(S\left(\underline{q}_{1}\right)+\lambda \underline{p}_{1} \underline{q}_{1}\right)+\alpha_{2} \underline{\mu}\left(S\left(\underline{q}_{2}\right)+\lambda \underline{p}_{2} \underline{q}_{2}\right)-(1+\lambda)\left(\alpha_{1} c_{1} \underline{q}_{1}+\alpha_{2} \underline{\mu}_{2} \underline{q}_{2}\right.\right. \\
& +C(\underline{\mu}))-\lambda \underline{U}\}+(1-\nu)\left\{\alpha _ { 1 } \left(S\left(\bar{q}_{1}+\lambda \bar{p}_{1} \bar{q}_{1}\right)+\alpha_{2} \bar{\mu}\left(S\left(\bar{q}_{2}\right)+\lambda \bar{p}_{2} \bar{q}_{2}\right)\right.\right. \\
& \left.-(1+\lambda)\left(\alpha_{1} c_{1} \bar{q}_{1}+\alpha_{2} \overline{\mu c}_{2} \bar{q}_{2}+C(\bar{\mu})\right)-\lambda \bar{U}\right\}
\end{array}
$$

s.t. (4) and (5).

Optimal regulation then entails: 
Proposition 1: Under complete information and discriminatory pricing the optimal prices follow the Ramsey rule and the optimal size of the network is such that the social marginal cost of network expansion is equal to the social marginal gain. More precisely,

$$
\begin{gathered}
\frac{\underline{p}_{1}^{*}-c_{1}}{\underline{p}_{1}^{*}}=\frac{\bar{p}_{1}^{*}-c_{1}}{\bar{p}_{1}^{*}}=\frac{\lambda}{1+\lambda} \frac{1}{\eta} \\
\frac{\underline{p}_{2}^{*}-\underline{c}_{2}}{\underline{p}_{2}^{*}}=\frac{\bar{p}_{2}^{*}-\bar{c}_{2}}{\bar{p}_{2}^{*}}=\frac{\lambda}{1+\lambda} \frac{1}{\eta} \\
(1+\lambda) C^{\prime}\left(\underline{\mu}^{*}\right)=\alpha_{2}\left[S\left(\underline{q}_{2}^{*}\right)+\lambda \underline{p}_{2}^{*} \underline{q}_{2}^{*}-(1+\lambda) \underline{c}_{2} \underline{q}_{2}^{*}\right] \\
(1+\lambda) C^{\prime}\left(\bar{\mu}^{*}\right)=\alpha_{2}\left[S\left(\bar{q}_{2}^{*}\right)+\lambda \bar{p}_{2}^{*} \bar{q}_{2}^{*}-(1+\lambda) \bar{c}_{2} \bar{q}_{2}^{*}\right] .
\end{gathered}
$$

Proof: See the Appendix.

Under complete information the regulator needs only to satisfy the participation constraints of the monopolistic firm so that no information rent is left to the firm. The optimal prices are set according to Ramsey principles. Mark-ups above marginal costs are due to the social cost of public funds.

To determine the optimal size of the network, one needs to take into account two types of benefits from providing services in the rural area. The net surplus effect, $S\left(\bar{q}_{2}\right)-(1+\lambda) \bar{c}_{2} \bar{q}_{2}$, represents the net benefits from consumption obtained by those connected to the rural network. The revenue effect, $\lambda \bar{p}_{2} \bar{q}_{2}$, is the social gain of revenues from those same consumers. Simple inspection of the first-order conditions shows that $\underline{p}_{1}^{*}=\bar{p}_{1}^{*}=p_{1}^{*}$ and $\underline{p}_{2}^{*}<\bar{p}_{2}^{*}$. Moreover, $\underline{\mu}^{*}>\bar{\mu}^{*}$. In other words, a higher marginal cost leads to a higher price and smaller network for the rural area.

By differentiating the network investment equation with respect to $\lambda$, one can find that the network size is a decreasing function of the social cost of public funds - that is, $\frac{d \mu^{*}}{d \lambda}<0$. So in a typical developing country, where $\lambda$ is larger than in industrial economies, network should be smaller from a normative point of view.

Now assume that the regulator has asymmetric information about $c_{2}$. Using the revelation principle, we can without loss of generality restrict our atten- 
tion to regulatory rules that are direct revelation mechanisms. Here a direct revelation mechanism is characterized by:

$$
\left\{p_{1}\left(\widetilde{c}_{2}\right), p_{2}\left(\widetilde{c}_{2}\right), \mu\left(\widetilde{c}_{2}\right), t\left(\widetilde{c}_{2}\right)\right\} \text { with } \widetilde{c}_{2} \in\left\{\underline{c}_{2}, \bar{c}_{2}\right\}
$$

where $\widetilde{c}_{2}$ is the monopolist's report to the government about its $c_{2}$.

For incentive compatibility, the following incentive constraints must be satisfied for the high-cost (bad-type) firm:

$$
\begin{array}{r}
\bar{t}+\alpha_{1} \bar{p}_{1} \bar{q}_{1}+\alpha_{2} \overline{\mu p}_{2} \bar{q}_{2}-\left(\alpha_{1} c_{1} \bar{q}_{1}+\alpha_{2} \overline{\mu c}_{2} \bar{q}_{2}\right)-C(\bar{\mu}) \\
\geq \quad \underline{t}+\alpha_{1} \underline{p}_{1} \underline{q}_{1}+\alpha_{2} \underline{\mu p}_{2} \underline{q}_{2}-\left(\alpha_{1} c_{1} \underline{q}_{1}+\alpha_{2} \underline{\mu}_{2} \underline{\bar{q}}_{2}\right)-C(\underline{\mu})
\end{array}
$$

and for the low-cost (good-type) firm:

$$
\begin{aligned}
& \underline{t}+\alpha_{1} \underline{p}_{1} \underline{q}_{1}+\alpha_{2} \underline{\mu p}_{2} \underline{q}_{2}-\left(\alpha_{1} c_{1} \underline{q}_{1}+\alpha_{2} \underline{\mu}_{2} \underline{q}_{2}\right)-C(\underline{\mu}) \\
\geq & \bar{t}+\alpha_{1} \bar{p}_{1} \bar{q}_{1}+\alpha_{2} \overline{\mu p}_{2} \bar{q}_{2}-\left(\alpha_{1} c_{1} \bar{q}_{1}+\alpha_{2} \underline{c}_{2} \overline{\mu q}_{2}\right)-C(\bar{\mu}) .
\end{aligned}
$$

Thus the government's program is:

$$
\begin{array}{cl}
\underset{\left\{\underline{q}_{1}, \underline{q}_{2}, \underline{\mu}, \underline{U}, \bar{q}_{1}, \bar{q}_{2}, \bar{\mu}, \bar{U}\right\}}{\ln } & \nu\left\{\alpha_{1}\left(S\left(\underline{q}_{1}\right)+\lambda \underline{p}_{1} \underline{q}_{1}\right)+\alpha_{2} \underline{\mu}\left(S\left(\underline{q}_{2}\right)+\lambda \underline{p}_{2} \underline{q}_{2}\right)-(1+\lambda)\left(\alpha_{1} c_{1} \underline{q}_{1}+\alpha_{2} \underline{\mu}_{2} \underline{c}_{2}\right.\right. \\
& +C(\underline{\mu}))-\lambda \underline{U})\}+(1-\nu)\left\{\alpha_{1}\left(S\left(\bar{q}_{1}\right)+\lambda \bar{p}_{1} \bar{q}_{1}\right)+\alpha_{2} \bar{\mu}\left(S\left(\bar{q}_{2}\right)+\lambda \bar{p}_{2} \bar{q}_{2}\right)\right. \\
& \left.-(1+\lambda)\left(\alpha_{1} c_{1} \bar{q}_{1}+\alpha_{2} \overline{\mu c}_{2} \bar{q}_{2}+C(\bar{\mu})\right)-\lambda \bar{U}\right\}
\end{array}
$$

s.t. (4), (5), (6), and (7).

That leads to the following result:

Proposition 2 : Under asymmetric information and discriminatory pricing the optimal regulatory policy entails:

(1) If $c_{2}=\underline{c}_{2}$, the optimal price and network size for the rural area are the same as under complete information;

(2) If $c_{2}=\bar{c}_{2}$, asymmetric information leads to a higher price and smaller network for the rural area than under complete information.

More specifically,

$$
\underline{p}_{1}^{S B}=\bar{p}_{1}^{S B}=p_{1}^{*}, \underline{p}_{2}^{S B}=\underline{p}_{2}^{*} ; \underline{\mu}^{S B}=\underline{\mu}^{*}
$$




$$
\begin{gathered}
\frac{\bar{p}_{2}^{S B}-\bar{c}_{2}}{\bar{p}_{2}^{S B}}=\frac{\lambda}{1+\lambda} \frac{1}{\eta}+\frac{\lambda}{1+\lambda} \frac{\nu}{1-\nu} \frac{\Delta c_{2}}{\bar{p}_{2}^{S B}} \\
(1+\lambda) C^{\prime}\left(\bar{\mu}^{S B}\right)=\alpha_{2}\left[S\left(\bar{q}_{2}^{S B}\right)+\lambda \bar{p}_{2}^{S B} \bar{q}_{2}^{S B}-(1+\lambda) \bar{c}_{2} \bar{q}_{2}^{S B}-\lambda \frac{\nu}{1-\nu} \Delta c_{2} \bar{q}_{2}^{S B}\right] .
\end{gathered}
$$

Proof: See the Appendix.

Under asymmetric information the regulator has to give up an information rent $\Pi=\alpha_{2} \bar{\mu} \Delta c_{2} \bar{q}_{2}$ to the efficient firm to satisfy incentive compatibility. Both the optimal price and the optimal network size for the rural area when $c_{2}=\bar{c}_{2}$ are distorted to mitigate this rent. Indeed, the optimal price in the rural area $\bar{p}_{2}$ is increased (consumption $\bar{q}_{2}$ decreased) and the optimal network size $\bar{\mu}_{2}$ decreased relative to the first-best allocations. Therefore, there are two effects distorting the optimal network size away from the first-best level if $\nu>0$ : (1) $\bar{q}_{2}$ is reduced, which leads to a reduction in $\bar{\mu}_{2}$; and $(2) \bar{\mu}_{2}$ itself is reduced to lower the rent.

Proposition 2 says that when price discrimination is allowed, under asymmetric information rural consumers face a higher price due to the information cost and so consume a lower level of services. Moreover, people without connections are less likely to be connected because the information rent increases the investment cost. Thus asymmetric information adversely affects both the connected and unconnected consumers in the rural area.

\section{Optimal Regulation Under Uniform Pricing}

This section derives the optimal regulatory policy under uniform pricing. As before, we first consider the benchmark case in which there is complete information about the cost of providing services in the rural area, $c_{2}$. For expositional simplicity, more notation is introduced: $\bar{p}=p\left(\bar{c}_{2}\right), \bar{q}=q(\bar{p}), \underline{p}=p\left(\underline{c}_{2}\right)$, and $q=q(p)$. Other notational conventions are defined as in the previous section.

Under complete information the following participation constraints must be satisfied for the high-cost (bad-type) firm:

$$
\bar{t}+\left(\alpha_{1}+\alpha_{2} \bar{\mu}\right) \overline{p q}-\left(\alpha_{1} c_{1}+\alpha_{2} \bar{c}_{2} \bar{\mu}\right) \bar{q}-C(\bar{\mu}) \geq 0,
$$


and for the low-cost (good-type) firm:

$$
\underline{t}+\left(\alpha_{1}+\alpha_{2} \underline{\mu}\right) \underline{p q}-\left(\alpha_{1} c_{1}+\alpha_{2} \underline{c}_{2} \underline{\mu}\right) \underline{q}-C(\underline{\mu}) \geq 0 .
$$

The government optimizes the following objective function:

$$
\begin{aligned}
\max _{(\underline{p}, \underline{\mu}, \bar{p}, \bar{\mu})} W & =\nu\left\{\left(\alpha_{1}+\alpha_{2} \underline{\mu}\right)(S(\underline{q})+\lambda \underline{p q})-(1+\lambda)\left[\left(\alpha_{1} c_{1}+\alpha_{2} \underline{\mu} \underline{c}_{2}\right) \underline{q}+C(\underline{\mu})\right]-\lambda \underline{U}\right\} \\
& +(1-\nu)\left\{\left(\alpha_{1}+\alpha_{2} \bar{\mu}\right)(S(\bar{q})+\lambda \overline{p q})-(1+\lambda)\left[\left(\alpha_{1} c_{1}+\alpha_{2} \overline{\mu c}_{2}\right) \bar{q}+C(\bar{\mu})\right]-\lambda \bar{U}\right\}
\end{aligned}
$$

subject to constraints (8) and (9).

Then optimal regulation entails:

Proposition 3 : Suppose that the government has complete information about $c_{2}$. Then uniform pricing reduces both the price and the network size for the rural area relative to those under price discrimination. More specifically,

$$
\begin{gathered}
\frac{\underline{p}^{* *}-\frac{\alpha_{1} c_{1}+\alpha_{2} \underline{c}_{2} \underline{\mu}^{* *}}{\alpha_{1}+\alpha_{2} \underline{\mu}^{* *}}}{\underline{p}^{* *}}=\frac{\bar{p}^{* *}-\frac{\alpha_{1} c_{1}+\alpha_{2} \bar{c}_{2} \bar{\mu}^{* *}}{\alpha_{1}+\alpha_{2} \bar{\mu}^{* *}}}{\bar{p}^{* *}}=\frac{\lambda}{1+\lambda} \frac{1}{\eta} \\
(1+\lambda) C^{\prime}\left(\underline{\mu}^{* *}\right)=\alpha_{2}\left[S\left(\underline{q}^{* *}\right)+\lambda \underline{p}^{* *} \underline{q}^{* *}-(1+\lambda) \underline{c}_{2} \underline{q}^{* *}\right] \\
(1+\lambda) C^{\prime}\left(\bar{\mu}^{* *}\right)=\alpha_{2}\left[S\left(\bar{q}^{* *}\right)+\lambda \bar{p}^{* *} \bar{q}^{* *}-(1+\lambda) \bar{c}_{2} \bar{q}^{* *}\right] .
\end{gathered}
$$

Proof: See the Appendix.

Under complete information the optimal allocation under uniform pricing can be implemented without giving up any information rent. Again, the prices are determined by the Ramsey rule. When the marginal cost in the rural area is $\underline{c}_{2}$ (resp. $\bar{c}_{2}$ ), the relevant cost is the average marginal cost $\frac{\alpha_{1} c_{1}+\alpha_{2} \underline{c}_{2} \underline{\underline{\mu}}}{\alpha_{1}+\alpha_{2} \underline{\underline{\mu}}}$ (resp. $\left.\frac{\alpha_{1} c_{1}+\alpha_{2} \bar{c}_{2} \bar{\mu}}{\alpha_{1}+\alpha_{2} \bar{\mu}}\right)$, which is lower than $\underline{c}_{2}$ (resp. $\left.\bar{c}_{2}\right)$ but higher than the marginal cost in the urban area, $c_{1}$. Thus uniform pricing leads to the redistributive outcome of a lower price for the rural area and a higher price for the urban area regardless of the network size - that is, $p_{1}^{*}<\underline{p}^{* *}<\underline{p}_{2}^{*}$ and $p_{1}^{*}<\bar{p}^{* *}<\bar{p}_{2}^{*}$. But as we will see soon, pricing favoritism towards the rural area is achieved with a distortion - resulting in a smaller network. 
When the monopolistic firm is endowed with a high-cost technology (that is $\left.c_{2}=\bar{c}_{2}\right), C^{\prime}(\bar{\mu})$ is the marginal cost of network expansion in the rural area. As before, two benefits of network expansion must be taken into account. One is the net surplus effect from consumption, $S(\bar{q})-(1+\lambda) \bar{q} \bar{c}_{2}$; the other is the revenue effect resulting from the social cost of public funds. But unlike under price discrimination, the net effect entails that $S(\bar{q})+\lambda \overline{p q}-(1+\lambda) \overline{q c}_{2}$ is a decreasing function of $\bar{q}$ at $\bar{q}^{* *}$ rather than a constant function. The reason is that uniform pricing creates a distortion resulting in a lower price in the rural area, which causes a distortion toward the size of the network. Thus $\underline{\mu}^{* *}<\underline{\mu}^{*}$ and $\bar{\mu}^{* *}<\bar{\mu}^{*}$.

A few remarks about uniform pricing are in order. Universal service obligations are often implemented using uniform pricing (such as for postal or telephone services). Although this regulatory policy may have political advantages, it is not necessarily the right approach to favor the rural area. For instance, the government could take a different approach to the design of its redistributive policy by changing the weights of consumers in different areas in the objective function. To illustrate this point, assume that the government puts a weight $\omega>1$ on the net consumer surplus in the rural area, which implies that it places a higher value on the surplus of these consumers. By simple manipulation the government's objective function can be written as:

$$
\begin{aligned}
W & =\alpha_{1}\left(S\left(q_{1}\right)-p_{1} q_{1}\right)+\alpha_{2} \mu \omega\left(S\left(q_{2}\right)-p_{2} q_{2}\right)-(1+\lambda) t+U \\
& =\alpha_{1}\left(S\left(q_{1}\right)+\lambda p_{1} q_{1}\right)+\alpha_{2} \mu \omega\left[S\left(q_{2}\right)+\left(\frac{1+\lambda}{\omega}-1\right) p_{2} q_{2}\right]-(1+\lambda)\left(\alpha_{1} c_{1} q_{1}+\alpha_{2} c_{2} \mu q_{2}+C(\mu)\right) .
\end{aligned}
$$

We thus obtain:

$$
\begin{aligned}
\frac{p_{2}^{\omega}-c_{2}}{p_{2}^{\omega}} & =\left(1-\frac{\omega}{1+\lambda}\right) \frac{1}{\eta} \\
(1+\lambda) C^{\prime}\left(\mu^{\omega}\right) & =\alpha_{2}\left[\omega\left(S\left(q_{2}^{\omega}\right)+\left(\frac{1+\lambda}{\omega}-1\right) p_{2}^{\omega} q_{2}^{\omega}\right)-(1+\lambda) c_{2} q_{2}^{\omega}\right] .
\end{aligned}
$$

To compare these results with those under uniform pricing, we substitute $p_{2}^{\omega}=\bar{p}^{* *}$ into the above pricing function to get $\omega=(1+\lambda)\left[1-\frac{\bar{p}^{* *}-\bar{c}_{2}}{\bar{p}^{* *}} \eta\right]$. One can show that $\omega\left(S\left(\bar{q}^{* *}\right)+\left(\frac{1+\lambda}{\omega}-1\right) \bar{p}^{* *} \bar{q}^{* *}-(1+\lambda) \bar{c}_{2} \bar{q}^{* *}>S\left(\bar{q}^{* *}\right)+\lambda \bar{p}^{* *} \bar{q}^{* *}-\right.$ 
$(1+\lambda) \bar{c}_{2} \bar{q}^{* *}{ }^{6}$ In other words, uniform pricing induces a smaller network with the same price for rural areas and achieves a lower level of social welfare with a weight, $\omega$, that induces the same price in rural areas. We can therefore conclude that, under complete information, favoring rural areas with proper price discrimination is a better way of implementing redistribution than using uniform pricing, if it is politically feasible. ${ }^{7}$

Assume now that the social planner has asymmetric information about $c_{2}$. The regulatory policy under uniform pricing (with $\omega=1$ ) can be defined as the following direct revelation mechanism:

$$
\left\{p\left(\widetilde{c}_{2}\right), \widetilde{\mu}\left(\widetilde{c}_{2}\right), t\left(\widetilde{c}_{2}\right)\right\} \text { with } \widetilde{c}_{2} \text { in }\left\{\underline{c}_{2}, \bar{c}_{2}\right\}
$$

Then the following incentive constraints need to be satisfied for the high-cost (bad-type) firm:

$\bar{t}+\left(\alpha_{1}+\alpha_{2} \bar{\mu}\right) \overline{p q}-\left(\alpha_{1} c_{1}+\alpha_{2} \bar{c}_{2} \bar{\mu}\right) \bar{q}-C(\bar{\mu}) \geq \underline{t}+\left(\alpha_{1}+\alpha_{2} \underline{\mu}\right) \underline{p q}-\left(\alpha_{1} c_{1}+\alpha_{2} \underline{\mu} \bar{c}_{2}\right) \underline{q}-C(\underline{\mu})$

and for the low-cost (good-type) firm:

$\underline{t}+\left(\alpha_{1}+\alpha_{2} \underline{\mu}\right) \underline{p q}-\left(\alpha_{1} c_{1}+\alpha_{2} \underline{c}_{2} \underline{\mu}\right) \underline{q}-C(\underline{\mu}) \geq \bar{t}+\left(\alpha_{1}+\alpha_{2} \bar{\mu}\right) \overline{p q}-\left(\alpha_{1} c_{1}+\alpha_{2} \bar{\mu} \underline{c}_{2}\right) \bar{q}-C(\bar{\mu})$.

The government needs to solve the following program for the optimal regulatory policy:

$$
\begin{array}{ll}
\underset{\underline{q}, \underline{\mu}, \underline{U}, \bar{q}, \bar{\mu}, \bar{U}\}}{\operatorname{Max}} & \nu\left\{\left(\alpha_{1}+\alpha_{2} \underline{\mu}\right)(S(\underline{q})+\lambda \underline{p q})-(1+\lambda)\left(\left(\alpha_{1} c_{1}+\alpha_{2} \underline{c}_{2} \underline{\mu}\right) \underline{q}+C(\underline{\mu})\right)-\lambda \underline{U}\right\} \\
& +(1-\nu)\left\{\left(\alpha_{1}+\alpha_{2} \bar{\mu}\right)(S(\bar{q})+\lambda \overline{p q})-(1+\lambda)\left[\left(\alpha_{1} c_{1}+\alpha_{2} \bar{c}_{2} \bar{\mu}\right) \bar{q}+C(\bar{\mu})\right]-\lambda \bar{U}\right\}
\end{array}
$$

s.t. (8), (9), (10), and (11).

We can now state the next result:

Proposition 4 : Suppose that the government has asymmetric information about $c_{2}$ and uses uniform pricing. Then:

\footnotetext{
${ }^{6}$ Since $\frac{\bar{p}^{* *}-\bar{c}_{2}}{\bar{p}^{* *}}-\frac{\lambda}{1+\lambda} \frac{1}{\eta}<0$, by collecting terms one has $\left(\frac{\lambda}{1+\lambda} \frac{1}{\eta}-\frac{\bar{p}^{* *}-\bar{c}_{2}}{\bar{p}^{* *}}\right)\left[S\left(\bar{q}^{* *}\right)-\bar{p}^{* *} \bar{q}^{* *}\right]>$ 0 .

${ }^{7}$ However, since our focus is on pricing as a way of redistribution, we remain the assumption of $\omega=1$ throughout the paper.
} 
(1) When the size of information asymmetry $\Delta c_{2}$ is large enough:

i) If $c_{2}=\underline{c}_{2}$, the optimal regulatory rule is the same as under complete information with uniform pricing; and

ii) If $c_{2}=\bar{c}_{2}$, the price is higher and the network size smaller in the rural area than under complete information with uniform pricing. Moreover, the price is lower and the network smaller than under asymmetric information with discriminatory pricing.

More specifically,

$$
\begin{gathered}
\underline{p}_{1}^{U S B}=p_{1}^{* *}, \underline{\mu}^{U S B}=\underline{\mu}^{* *} \\
\frac{\bar{p}^{U S B}-\frac{\alpha_{1} c_{1}+\alpha_{2} \bar{c}_{2} \bar{\mu}^{U S B}}{\alpha_{1}+\alpha_{2} \bar{\mu}^{U S B}}}{\bar{p}^{U S B}}=\frac{\lambda}{1+\lambda} \frac{1}{\eta}+\frac{\lambda}{1+\lambda} \frac{\nu}{1-\nu} \frac{\alpha_{2} \Delta c_{2} \bar{\mu}^{U S B}}{\alpha_{1}+\alpha_{2} \bar{\mu}^{U S B}} \frac{1}{\bar{p}^{U S B}} \\
(1+\lambda) C^{\prime}\left(\bar{\mu}^{U S B}\right)=\alpha_{2}\left[S\left(\bar{q}^{U S B}\right)+\lambda \bar{p}^{U S B} \bar{q}^{U S B}-(1+\lambda) \bar{c}_{2} \bar{q}^{U S B}-\lambda \frac{\nu}{1-\nu} \Delta c_{2} \bar{q}^{U S B}\right] ;
\end{gathered}
$$

(2) When the size of information asymmetry $\Delta c_{2}$ is small enough, bunching occurs and optimal regulation entails:

$$
\begin{gathered}
\frac{p^{U S B}-\frac{\alpha_{1} c_{1}+\alpha_{2}\left[\nu \underline{c}_{2}+(1-\nu) \bar{c}_{2}\right] \mu^{U S B}}{\alpha_{1}+\alpha_{2} \mu^{U S B}}}{p^{U S B}}=\frac{\lambda}{1+\lambda} \frac{1}{\eta}+\frac{\lambda}{1+\lambda} \frac{\nu \alpha_{2} \Delta c_{2} \mu^{U S B}}{\alpha_{1}+\alpha_{2} \mu^{U S B}} \frac{1}{p^{U S B}} \\
C^{\prime}\left(\mu^{U S B}\right)=\alpha_{2}\left[\frac{S\left(q^{U S B}\right)+\lambda p^{U S B} q^{U S B}}{1+\lambda}-\left(\nu \underline{c}_{2}+(1-\nu) \bar{c}_{2}\right) q^{U S B}-\frac{\lambda}{1+\lambda} \nu \Delta c_{2} q^{U S B}\right] .
\end{gathered}
$$

Proof: See the Appendix.

Under uniform pricing and asymmetric information, the optimal regulatory rule is obtained when $c_{2}=\underline{c}_{2}$-that is, there is no distortion at top. But since the government has to give up an information rent, $\alpha_{2} \bar{\mu} \Delta c_{2} \bar{q}$, to the firm in order to induce truthful revelation of its information, both the price and the network size are distorted as a result of the efficiency-rent-extraction trade-off when the firm has a high-cost technology.

Uniform pricing under asymmetric information has two effects on the pricing decision. One is the direct effect of asymmetric information, which leads to a higher price. The other is the average marginal cost effect caused by uniform 
pricing, which tends to induce a lower price. The net effect is in general ambiguous because the first-order conditions are simultaneous equations of $\bar{p}$ and $\bar{\mu}$. But if the size of information asymmetry $\Delta c_{2}$ is large enough, under uniform pricing the price for the rural area is higher than under complete information.

To determine the optimal network investment, one needs now to take into account three effects: In addition to the surplus effect and the revenue effect, there is a direct effect of asymmetric information that calls for a reduction of the network size. The net effect is to induce a smaller network if $\Delta c_{2}$ is large enough.

Next we compare these results with those under discriminatory pricing. There are two effects that need to be considered. In addition to the average marginal cost effect, which leads to a decrease in price, there is another effect which, by reducing information cost, also induces a lower price. Therefore, the price under uniform pricing is always lower than under discriminatory pricing. More specifically, denote $\overline{\bar{p}} \equiv \frac{\alpha_{1} p_{1}+\alpha_{2} \overline{\mu p_{2}}}{\alpha_{1}+\alpha_{2} \bar{\mu}}$ as the average price under price discrimination, one can obtain that the price under uniform pricing is equal to the average price under discrimination $\left(\overline{\bar{p}}=\bar{p}^{U S B}\right)$ for a given size of the network. As a result the size of the network under uniform pricing is smaller than under discriminatory pricing. We thus conclude that, while under asymmetric information, uniform pricing does favor the rural people contrary to what was observed under full information, it does so at the expense of network expansion, therefore penalizing unconnected users (as well as future users).

An interesting additional result under uniform pricing is that bunching occurs if $\Delta c_{2}$ is small enough. In that case optimization of the social welfare calls for consumption and network investment to be decreasing functions of $c_{2} \cdot{ }^{8}$ But in the presence of asymmetric information, if $\Delta c_{2}$ is small enough, the second-order condition of truth-telling requires both the consumption and the network size be increasing functions of $c_{2} \cdot{ }^{9}$ These opposite monotonic conditions required by the optimization of the objective function and the imple-

\footnotetext{
${ }^{8}$ Note that in the optimal allocation under complete information, monotonicity is satisfied if $\alpha_{1}$ is large enough.

${ }^{9}$ Here the action space is two dimensional.
} 
mentability of allocations or by satisfying incentive compatibility lead to the non-responsiveness result. That is, screening is no longer possible with uniform pricing. ${ }^{10}$

\section{Universal Service Policy Under Collusion}

We now consider the possibility of collusion, to illustrate in a very simple setting the impact of collusion on the optimal regulatory policy. For this purpose we add to the basic principal-agent relationship between the government and the monopolist firm a hierarchical level representing the regulator of the firm. We also consider other interest groups which have stakes in regulation. Indeed, a special feature of this paper is that in addition to the threat of collusion between the regulator and the firm, collusion may arise between the regulator and other interest groups such as taxpayers and rural consumers.

Following Tirole (1986), we assume that the regulator's role is to bridge the government's information gap about $c_{2}$. Suppose the regulator has utility function:

$$
R(s)=s \text { and } s \geq 0
$$

where $s$ is the regulator's reward. The regulator is risk neutral but is protected by limited liability. Therefore, to obtain the regulator's participation he should get at least his reservation utility level, which is normalized to be zero.

Assume that the regulator is endowed with an information technology that he uses to obtain a private signal $\left(\sigma=c_{2}\right)$ about the monopolist's cost in the rural area with probability $\xi$ and learns nothing $(\sigma=\phi)$ with probability $1-\xi$. For simplicity we assume that the regulator's information is known to the monopolistic firm and other possible interest groups. In other words, sidecontracting is assumed to take place under complete information. ${ }^{11}$ To make use of the regulator's information $\sigma$, the government asks him to report the

\footnotetext{
${ }^{10}$ For an exposition of this non responsiveness result in a model with a single action see Laffont and Martimort (2002, p. 53).

${ }^{11}$ One can think of the principal as the public opinion and the regulator is an independent regulator. Thus it is possible that many people such as the firm, urban and rural users, and taxpayers are informed but the government is not.
} 
signal he has received - that is, $r \in\left\{\underline{c}_{2}, \bar{c}_{2}, \phi\right\}$. The critical assumption is that the signal the regulator reports to the government is hard information - when a signal is reported to the government, it is hard evidence. But the regulator can hide information and report that the signal is $\phi$. If the regulator learns nothing, he must report $\sigma=\phi$. Thus the regulator has discretion only when he receives a signal that reveals the firm's private information. The regulator's information technology is summarized in the following table describing the probability of each state of nature.

\begin{tabular}{|l|l|l|}
\hline & type $\bar{c}_{2}$ & type $\underline{c}_{2}$ \\
\hline$\sigma=\bar{c}_{2}$ & $\nu \xi$ & 0 \\
\hline$\sigma=\phi$ & $\nu(1-\xi)$ & $(1-\nu)(1-\xi)$ \\
\hline$\sigma=\underline{c}_{2}$ & 0 & $(1-\nu) \xi$ \\
\hline
\end{tabular}

The timing of the regulatory game in the presence of a collusion threat between the regulator and the monopolist and other interest groups is as follows. The government offers a grand contract after the regulator, the firm, and other interest groups learn their respective information ${ }^{12}$. The regulator then makes a take-it-or-leave-it offer to the firm and other interest groups and they decide whether to accept this side-contract. The government then asks the regulator for a report about the monopolist's cost. When evidence is reported by the regulator, the regulatory contract is chosen under complete information. But when the regulator reports that he has learned nothing, the revelation game under asymmetric information is played as in the previous section. Finally, the firm decides whether to accept the grand contract, and both the grand contract and the side contract will be executed if they are accepted. ${ }^{13}$

To simplify exposition, the following notation is introduced: $\bar{q}_{2}^{c}=q\left(c_{2}=\right.$

\footnotetext{
${ }^{12}$ One may be worried about the possibility of Maskin games (Maskin, 1999) — that is, pushing one group against another to verify information. Indeed, there might exist a more general class of mechanisms (perhaps bizarre) such as asking urban users to predict what the regulator would announce and give a small discount if they get it right-and use this information to regulate. However, as is standard in the literature, we will be restricting attention to mechanisms in which $\sigma$ is announced by the regulator and $c_{2}$ by the firm.

${ }^{13}$ Note that under our assumption about the regulator's information technology, the posterior beliefs after an inconclusive signal $(\emptyset)$ are identical to the prior beliefs:

$$
\hat{\nu}=\frac{\nu(1-\xi)}{\nu(1-\xi)+(1-\nu)(1-\xi)}=\nu .
$$
}


$\left.\bar{c}_{2}, \sigma=\bar{c}_{2}\right), \underline{q}_{2}^{c}=q\left(c_{2}=\underline{c}_{2}, \sigma=\underline{c}_{2}\right), \bar{q}_{2}^{c \phi}=q\left(c_{2}=\bar{c}_{2}, \sigma=\phi\right)$, and $\underline{q}_{2}^{c \phi}=q\left(c_{2}=\right.$ $\left.\underline{c}_{2}, \sigma=\phi\right)$. Other notation such as $\underline{\mu}^{c}, \underline{\mu}^{c \phi}, \bar{\mu}^{c}$, and $\bar{\mu}^{c \phi}$ is similarly defined.

To analyze when collusion matters, assume that the second-best contract under discriminatory pricing is still offered under the threat of collusion. Let us look first at what happens if the state of nature is $c_{2}=\underline{c}_{2}$ and $\sigma=\underline{c}_{2}$. In this case the firm gets an information rent of $\alpha_{2} \bar{\mu}^{S B} \Delta c_{2} \bar{q}_{2}^{S B}$ if the regulator hides the information that the firm is efficient. So there is scope for collusion-the good-type firm may bribe the regulator for his silence. Thus the government has to satisfy the following coalition incentive constraint to induce the regulator to tell the truth:

$$
\underline{s}^{f} \geqslant k_{1} \alpha_{2} \Delta c_{2} \bar{\mu}^{c \phi} \bar{q}_{2}^{c \phi},
$$

where $\underline{s}^{f}$ is the reward of the regulator if he reveals that the firm is type $\underline{c}_{2}$, and $k_{1}=\frac{1}{1+\lambda_{1}}$ where $\lambda_{1}$ represents the transaction cost between the regulator and the firm. To focus on the interesting case we assume $\lambda_{1}>\lambda .{ }^{14}$ Extending Proposition 2 to take into account the cost of collusion-proofness, we know that for $c_{2}=\underline{c}_{2}$ the price and the network size are not distorted regardless of the regulator's report. Thus consumers in the rural area are not affected by the presence of collusion in this state of nature. Similarly, urban consumers will not collude with the regulator because of complete information about $c_{1}$. Another potential interest group is taxpayers, who are obliged to fund any deficit due to the lack of budget constraint for the firm. Nevertheless, because neither the price nor the network size are distorted by the presence of asymmetric information, taxpayers will pay the same amount of taxes under asymmetric information as under complete information. So, taxpayers do not want to collude with the regulator either. Thus $\underline{s}^{f}>0$ is the only incentive payment to the regulator to induce his truthful report.

Suppose the state of nature is $c_{2}=\bar{c}_{2}$ and $\sigma=\bar{c}_{2}$. In this case the firm does not obtain any information rent even if the regulator hides the evidence. So, the monopolistic firm does not want to collude with the regulator. Similarly, urban consumers do not collude with the regulator. Moreover, we know from

\footnotetext{
${ }^{14}$ Otherwise, side-contracting incurs a lower transaction cost than the social cost of public funds and it is optimal to let collusion take place. So avoiding collusion is not an issue.
} 
Proposition 2 that rural consumers would get a surplus of $\alpha_{2} \bar{\mu}^{S B}\left(S\left(\bar{q}_{2}^{S B}\right)-\right.$ $\left.\bar{p}_{2}^{S B} \bar{q}_{2}^{S B}\right)$ if the signal were concealed by the regulator, and $\alpha_{2} \bar{\mu}^{*}\left(S\left(\bar{q}_{2}^{*}\right)-\bar{p}_{2}^{*} \bar{q}_{2}^{*}\right)$ if the signal were truthfully revealed. Since asymmetric information leads to a higher price and smaller network for the rural area under price discrimination and $S(q)-p q$ is an increasing function of $q$ in the relevant region, rural consumers would obtain less surplus from collusion and so do not have any incentive to collude with the regulator.

For taxpayers, however, if $\sigma=\bar{c}_{2}$ is reported to the government, they will pay:

$$
T^{c}=\alpha_{1} \bar{q}_{1}^{*}\left(c_{1}-\bar{p}_{1}^{*}\right)+\alpha_{2} \bar{\mu}^{*} \bar{q}_{2}^{*}\left(\bar{c}_{2}-\bar{p}_{2}^{*}\right)+C\left(\bar{\mu}^{*}\right)
$$

and

$$
T^{c \phi}=\alpha_{1} \bar{q}_{1}^{S B}\left(c_{1}-\bar{p}_{1}^{S B}\right)+\alpha_{2} \bar{\mu}^{S B} \bar{q}_{2}^{S B}\left(\bar{c}_{2}-\bar{p}_{2}^{S B}\right)+C\left(\bar{\mu}^{S B}\right)
$$

if $\sigma$ is hidden. Since asymmetric information induces a higher price and smaller network in the rural area, one can check that $T^{c \phi}<T^{c}$. In other words, taxpayers pay less to fund the deficit if the regulator hides the information that $\sigma=\bar{c}_{2}$. Thus taxpayers want to collude with the regulator if they can overcome the organizational costs involved and ask the regulator to hide the signal $\sigma=\bar{c}_{2}$ in order to save a tax payment of $T^{c}-T^{\phi}$. In this case the government has to satisfy the following coalition incentive constraint:

$$
\bar{s}^{t} \geqslant k_{2}\left(T^{c}-T^{c \phi}\right)
$$

where $k_{2}=\frac{1}{1+\lambda_{2}}$ and $\lambda_{2}$ denotes the transaction cost of side-contracting between the regulator and taxpayers. Again, we assume $\lambda_{2}>\lambda$ to focus on the interesting case where collusion is an issue. Assume also that the government's program remains concave.

Let $W^{F I}\left(q_{1}^{c}, q_{2}^{c}, \mu^{c}\right)$ and $W^{F I}\left(q_{1}^{c \phi}, q_{2}^{c \phi}, \mu^{c \phi}\right)$ denote the expected welfare under full information and under asymmetric information, respectively. The government optimizes the following program to solve for the optimal regulatory policy under the threat of collusion:

$$
\begin{array}{cc}
\operatorname{Max}_{\left\{\underline{q}_{1}^{c}, \underline{q}_{2}^{c}, \underline{q}_{2}^{c \phi}, \underline{\mu}^{c}, \underline{\mu}^{c \phi}, \bar{q}_{1}^{c}, \bar{q}_{2}^{c}, \bar{q}_{2}^{c \phi}, \bar{\mu}^{c}, \bar{\mu}^{c \phi}\right\}} & \left\{\xi W^{F I}\left(q_{1}^{c}, q_{2}^{c}, \mu^{c}\right)+(1-\xi) W^{F I}\left(q_{1}^{c \phi}, q_{2}^{c \phi}, \mu^{c \phi}\right)\right. \\
& \left.-\xi \nu \lambda k_{1} \alpha_{2} \Delta c_{2} \bar{\mu}^{c \phi} \bar{q}_{2}^{c \phi}-\xi(1-\nu) \lambda k_{2}\left(T^{c}-T^{c \phi}\right)\right\}
\end{array}
$$


subject to constraints (7),(8),(9),(10),(13), and (14).

Note that when the state of nature is $c_{2}=\underline{c}_{2}$ and $\sigma=\underline{c}_{2}$, the government does not need to give up any information rent but has to offer an incentive payment, $k_{1} \alpha_{2} \Delta c_{2} \bar{\mu}^{c \phi} \bar{q}_{2}^{c \phi}$, to the regulator in order to induce truthful revelation of information. In that case regulation is made under complete information. When the state of nature is $c_{2}=\underline{c}_{2}$ and $\sigma=\phi$, regulatory policies are made under asymmetric information. In this case the government not only has to pay the regulator's reservation utility level but has to give up an information rent, $\alpha_{2} \Delta c_{2} \bar{\mu}^{c \phi} \bar{q}_{2}^{c \phi}$, to the good-type firm.

When the state of nature is $c_{2}=\bar{c}_{2}$ and $\sigma=\bar{c}_{2}$, the monopolistic firm does not get any information rent. But the regulator receives an incentive payment of $\bar{s}^{t}=\frac{1}{1+\lambda_{2}}\left(T^{c}-T^{c \phi}\right)$ to reveal his information.

We thus obtain the following result:

Proposition 5: Assume that the government has asymmetric information about $c_{2}$ and uses discriminatory pricing ${ }^{15}$. Then the optimal regulatory response to the threat of collusion calls for:

i) When $c_{2}=\underline{c}_{2}$, the price and the network size in the rural area are the same as first-best allocations;

ii) When the state of nature is $c_{2}=\bar{c}_{2}$ and $\sigma=\phi$, The regulatory response is to reduce not only the information rent available to the firm but also the tax savings to taxpayers. The optimal size of the rural network is smaller than without the threat of collusion. Moreover, the optimal price for the rural area is higher than in the absence of collusion if $k_{1}>\frac{\lambda}{1+\lambda}\left(1-\frac{1}{\eta}\right) k_{2} /\left(1-\frac{\lambda}{1+\lambda} \frac{1}{\eta}\right)$.

iii) When the state of nature is $c_{2}=\bar{c}_{2}$ and $\sigma=\bar{c}_{2}$, The regulatory response is to reduce the tax savings available to taxpayers. The optimal price for the rural area is higher and the optimal size of the rural network is smaller than in the absence of collusion.

\footnotetext{
${ }^{15}$ For simplicity we also assume that the second-order condition for thuth-telling is satisfied. Otherwise, bunching occurs and collusion leads to less screening.
} 
More specifically, the optimal regulatory policy entails:

$$
\begin{aligned}
& \underline{p}_{2}^{c}=\underline{p}_{2}^{c \phi}=\underline{p}_{2}^{*}, \underline{\mu}^{c}=\underline{\mu}^{c \phi}=\underline{\mu}^{*} \\
& \frac{\bar{p}_{2}^{c \phi}-\bar{c}_{2}}{\bar{p}_{2}^{c \phi}}\left[1+\frac{\lambda}{1+\lambda} \frac{\xi}{1-\xi} k_{2}\right]=\frac{\lambda}{1+\lambda} \frac{1}{1-\xi} \frac{1}{\eta}\left[\xi k_{2}+(1-\xi)\right] \\
& +\frac{\lambda}{1+\lambda} \frac{\nu}{1-\nu} \frac{1}{1-\xi} \frac{\Delta c_{2}}{\bar{p}_{2}^{c \phi}}\left[\xi k_{1}+(1-\xi)\right] \\
& \frac{\bar{p}_{2}^{c}-\bar{c}_{2}}{\bar{p}_{2}^{c}}\left(1+\frac{\lambda}{1+\lambda} k_{2}\right)=\frac{\lambda}{1+\lambda} \frac{1}{\eta}\left(1+k_{2}\right) \\
& C^{\prime}\left(\bar{\mu}^{c \phi}\right)\left[(1+\lambda)-\lambda \frac{\xi}{1-\xi} k_{2}\right]=\alpha_{2}\left[S\left(\bar{q}_{2}^{c \phi}\right)+\lambda \bar{p}_{2}^{c \phi} \bar{q}_{2}^{c \phi}-(1+\lambda) \bar{c}_{2} \bar{q}_{2}^{c \phi}\right. \\
& -\lambda \frac{\nu}{1-\nu} \frac{\bar{q}_{2}^{c \phi} \Delta c_{2}}{1-\xi}\left(k_{1} \xi+(1-\xi)\right)-\lambda \frac{\xi}{1-\xi} k_{2} \bar{q}_{2}^{c \phi}\left(\bar{p}_{2}^{c \phi}-\bar{c}_{2}\right) \\
& C^{\prime}\left(\bar{\mu}^{c}\right)\left[(1+\lambda)+\lambda k_{2}\right]=\alpha_{2}\left[S\left(\bar{q}_{2}^{c}\right)+\lambda \bar{p}_{2}^{c} \bar{q}_{2}^{c}-(1+\lambda) \bar{c}_{2} \bar{q}_{2}^{c}+\lambda k_{2} \bar{q}_{2}^{c}\left(\bar{p}_{2}^{c}-\bar{c}_{2}\right)\right] .
\end{aligned}
$$

Proof: The proof of Proposition 5 is straightforward so it is omitted.

Under our assumptions the binding constraints are the participation constraint of the bad-type, the incentive constraint of the good-type, and the collusion-proof constraints (13) and (14). When the state of nature is $c_{2}=\underline{c}_{2}$ and $\sigma=\underline{c}_{2}$, since the good-type firm obtains an information rent of $\alpha_{2} \Delta c_{2} \bar{\mu}^{c \phi} \bar{q}_{2}^{c \phi}$ if the regulator reports $r=\phi$, the optimal regulatory response to the threat of collusion between the firm and the regulator is to raise the price $\bar{p}_{2}^{c \phi}$ and reduce the network size $\bar{\mu}^{c \phi}$. In other words, incentives given to the firm are weakened to reduce the stake of collusion.

But when the state of nature is $c_{2}=\bar{c}_{2}$ and $\sigma=\bar{c}_{2}$, taxpayers receives a tax saving of $T^{c}-T^{c \phi}$ if the regulator conceals his information. In this case prevention of collusion requires lowering the price $\bar{p}_{2}^{c \phi}$ and raising the network size $\bar{\mu}^{c \phi}$ on the one hand, and raising the price $\bar{p}_{2}^{c}$ and reducing the network size $\bar{\mu}^{c}$ on the other, to reduce taxpayers' incentives in regulation. Since both incentive payments to the regulator, $\underline{s}^{f}$ and $\bar{s}^{t}$, depend on the output $\bar{q}_{2}^{c \phi}$, we have an interesting case where, from the government's point of view, the monopolistic firm and taxpayers have similar incentives to collude with the regulator-both have stakes in inefficient regulation. As a result the existence of one coalition incentive constraint hardens the other one, even though these coalition constraints are associated with different states of nature. Indeed, satisfying the coalition 
incentive constraint between the regulator and taxpayers requires that the incentive for taxpayers are weakened ( $\bar{\mu}^{c \phi}$ and $\bar{q}_{2}^{c \phi}$ are increased); but raising $\bar{\mu}^{c \phi}$ and $\bar{q}_{2}^{c \phi}$ strengthens the incentives offered to the firm which are socially costly. However, it turns out that the need to reduce the stake of collusion between the regulator and the firm dominates that between the regulator and taxpayers if $k_{1}$ is large or $\lambda_{1}$ small. The intuition is simple -if the transaction cost between the regulator and the firm is small, the collusion threat is then more likely to be an issue than that between the regulator and taxpayers. Consequently, the price in the rural area will be higher than without collusion. Conversely, the presence of collusion induces a lower price for the rural area if $k_{1}$ is small. ${ }^{16}$

At this stage one may wonder whether the result about collusion under discriminatory pricing also holds under uniform pricing. We show this robustness result in the appendix and limit ourselves only with collecting the result below.

Proposition 6 : Assume that the government has asymmetric information about $c_{2}$ and uses uniform pricing. Then the optimal regulatory response to the threat of collusion entails:

(1) When $c_{2}=\underline{c}_{2}$, the allocations are the same as under complete information;

(2) When the state of nature is $c_{2}=\bar{c}_{2}$ and $\sigma=\phi$ :

i) The price for the rural area is lower and the size of network is smaller than under discriminatory pricing, and

ii) If $k_{1}$ is large, the price for the rural area is higher and the network size smaller than in the absence of collusion;

(2) When $c_{2}=\bar{c}_{2}$ and $\sigma=\bar{c}_{2}$, the price is higher and the network size smaller in the rural area than in the absence of collusion.

Proof: See the Appendix.

\footnotetext{
${ }^{16}$ It is interesting to note that this result is similar to Laffont and Tirole (1993, p. 490) where the existence of a different interest group (environmentalists) increases the firm's rent. In both cases the firm and taxpayers have a stake in making regulation inefficient.
} 
As for the case of discriminatory pricing, the monopolistic firm's incentives are weakened by the existence of taxpayers as an interest group. Moreover, allocations are distorted when $c_{2}=\bar{c}_{2}$ and the regulator learns this information and then reports it to the government. But in both states of nature $c_{2}=\bar{c}_{2}$ and $\sigma=\phi$ and $c_{2}=\bar{c}_{2}$ and $\sigma=\bar{c}_{2}$, the price for the rural area is lower and the size of the network is smaller than those under discriminatory pricing.

\section{Conclusion}

This paper has built a formal model to analyze the optimal regulatory policy associated with universal service obligations. In particular, it has examined how optimal universal service policy is affected by asymmetric information and the threat of regulatory capture under both discriminatory pricing and uniform pricing. The findings show that under both types of pricing, asymmetric information leads to a higher price and smaller network in the rural area and the collusion threat weakens the incentives given to the service-providing firm. In addition, although uniform pricing may achieve the redistributive goal of a lower price for the rural area, it is at the cost of a smaller network.

One of the main policy implications of these results is that when uniform pricing is used to favor rural consumers, consumers who are not connected may be adversely affected because less investment is directed towards network expansion. Thus uniform pricing may not achieve the goal claimed by policy makers of promoting increased access to infrastructure services - an urgent need in developing countries. On the contrary, network expansion may be delayed precisely because rural consumers are favored in pricing. Thus one general lesson from this paper is that one cannot isolate the network investment problem from pricing policy.

Because developing countries have not yet developed sufficient infrastructure networks to provide services to all members of society, network expansion is an extremely important development strategy for these countries. Indeed, in many developing countries poor people are willing to pay a high price to access to 
basic infrastructure services. But since there is no network in place, they are deprived of the services. Thus governments in developing countries should pay more attention to providing incentives for network investments rather than to distorting prices.

Another contribution of the paper is to provide a better description of the losers and winners in the universal service policy game. Furthermore, as the agency-based theory of capture has shown, having a stake is a necessary but not sufficient condition for an interest group to potentially be able to capture the regulator. Accordingly, we have characterized the optimal regulatory responses. Ultimately, one of the main results may be that the universal service policy is easily manipulated.

\section{Acknowledgements}

We thank especially Jean Tirole for his very detailed comments and suggestions and the participants at the IO workshop at Guanghua Management School of Beijing University for their comments. 


\section{References}

Chisari, O., Estache, A., Waddams-Price, C., 2003. Access by the Poor in Latin America's Utility Reform: Subsidies and Service Obligations. In: Ugaz, C., Waddams-Price, C. (Eds), Utility Privatization and Regulation: A Fair Deal for Consumers?. Edward Elgar, Northampton, MAS, USA.

Clark, G., Wallsten, S., 2002. Universal(ly Bad) Service: Providing Infrastructure Services to Rural and Poor Urban Consumers. World Bank, Policy Research Working Paper 2868.

Cremer, H., Gasmi, F., Grimaud, A., Laffont, J.-J., 2001. Universal Service: An Economic Perspective. Annals of Public and Cooperative Economics 71, $5-42$.

Estache, A., Laffont, J.-J., Zhang, X.-Z., 2003. Universal Service Obligations in Developing Countries. Working Paper, Research Center for Regulation and Competition, Beijing.

Gasmi, F., Laffont, J.-J., Sharkey, W., 1999. Competition, Universal Service and Telecommunications Policy in Developing Countries. Information Economics and Policy 12, 221-248.

Guesnerie, R., Laffont, J.-J., 1984. A Complete Solution to a Class of Principal-Agent Problems with an Application to the Control of Self-Managed Firm. Journal of Public Economics 25, 329-369.

Laffont, J.-J., N'Gbo, A., 2000. Cross-Subsidies and Network Expansion in Developing Countries. European Economic Review 44, 797-805.

Laffont, J.-J., Tirole, J., 1993. A Theory of Incentives in Procurement and Regulation. MIT Press, Cambridge. 
Laffont, J.-J., Tirole, J., 2002. Competition in Telecommunications. MIT Press, Cambridge.

Laffont, J.-J., Martimort, D., 2002. The Theory of Incentives I: The PrincipleAgent Model. Princeton University Press, Princeton.

Maskin, E., 1999. Nash Equilibrium and Welfare Optimality. Review of Economic Studies 66, 23-38.

Tirole, J., 1986. Hierarchies and Bureaucracies. Journal of Law, Economics, and Organization 2, 181-214. 


\section{Appendix}

\section{Proof of Proposition 1:}

The first-order conditions with respect to $\bar{q}_{1}, \bar{q}_{2}, \bar{\mu}$ (and similarly for those with respect to $\left.\underline{q}_{1}, \underline{q}_{2}, \underline{\mu}\right)$ are:

$$
\begin{aligned}
\alpha_{1}\left(S^{\prime}\left(\bar{q}_{1}\right)+\lambda \bar{p}_{1}^{\prime} \bar{q}_{1}+\lambda \bar{p}_{1}\right)-(1+\lambda) \alpha_{1} c_{1} & =0 \\
\alpha_{2} \bar{\mu}\left(S^{\prime}\left(\bar{q}_{2}\right)+\lambda \bar{p}_{2}^{\prime} \bar{q}_{2}+\lambda \bar{p}_{2}\right)-(1+\lambda) \alpha_{2} \bar{\mu} \bar{c}_{2} & =0 \\
\alpha_{2}\left(S\left(\bar{q}_{2}\right)+\lambda \bar{p}_{2} \bar{q}_{2}\right)-(1+\lambda)\left(\alpha_{2} \bar{c}_{2} \bar{q}_{2}+C^{\prime}(\bar{\mu})\right) & =0 .
\end{aligned}
$$

Since $C^{\prime \prime}>0$, a sufficient condition for the concavity of the objective function is $\left|S^{\prime \prime}\right|$ large enough.

The determination of $\bar{p}_{2}$ and $\bar{\mu}$ is illustrated in Figure 1.

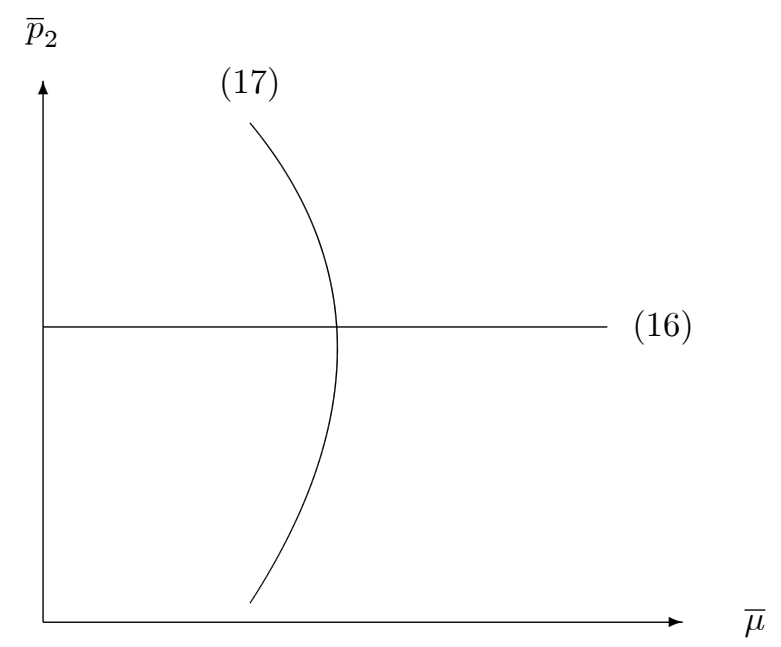

Figure 1

Equation (16) determines $\bar{q}_{2}$ according to the Ramsey rule. ${ }^{17}$ For this level

${ }^{17}$ There is of course an one to one relationship between $\bar{p}_{2}$ and $\bar{q}_{2}$. 
of $\bar{q}_{2},(17)$ determines $\bar{\mu}$.

\section{Proof of Proposition 2:}

Since only $\bar{q}_{2}$ and $\bar{\mu}$ are affected by asymmetric information, we only consider the first-order conditions with respect to $\bar{q}_{2}$ and $\bar{\mu}$ :

$$
\begin{aligned}
-\nu \lambda \alpha_{2} \Delta c_{2} \bar{\mu}+(1-\nu)\left[\alpha_{2}\left(S^{\prime}\left(\bar{q}_{2}\right)+\lambda \bar{p}_{2}^{\prime} \bar{q}_{2}+\lambda \bar{p}_{2}\right)-(1+\lambda) \alpha_{2} \bar{c}_{2}\right] \bar{\mu} & =(\text { 08) } \\
-\nu \lambda \alpha_{2} \Delta c_{2} \bar{q}_{2}+(1-\nu)\left[\alpha_{2}\left(S\left(\bar{q}_{2}\right)+\lambda \bar{p}_{2} \bar{q}_{2}\right)-(1+\lambda)\left(\alpha_{2} \bar{c}_{2} \bar{q}_{2}+C^{\prime}(\bar{\mu})\right]\right. & =\text { (09) }
\end{aligned}
$$

$\left|S^{\prime \prime}\right|$ large enough ensures concavity.

Denote

$$
\begin{gathered}
U\left(c_{2}, \widetilde{c}_{2}\right) \equiv t\left(\widetilde{c}_{2}\right)+\alpha_{1} p_{1}\left(\widetilde{c}_{2}\right) q\left(p_{1}\left(\widetilde{c}_{2}\right)\right)+\alpha_{2} \mu\left(\widetilde{c}_{2}\right) p_{2}\left(\widetilde{c}_{2}\right) q\left(p_{2}\left(\widetilde{c}_{2}\right)\right) \\
-\left(\alpha_{1} c_{1} q\left(p_{1}\left(\widetilde{c}_{2}\right)\right)+\alpha_{2} c_{2} \mu\left(\widetilde{c}_{2}\right) q\left(p_{2}\left(\widetilde{c}_{2}\right)\right)\right)-C\left(\mu\left(\widetilde{c}_{2}\right)\right)
\end{gathered}
$$

as the utility the firm obtains when its report about $c_{2}$ is $\widetilde{c}_{2}$. Proceeding as if $c_{2}$ was a continuous variable, the first-order condition for truth-telling is:

$$
\begin{gathered}
\dot{t}+\alpha_{1}\left(\dot{p}_{1} q_{1}^{\prime} p_{1}+\dot{p}_{1} q_{1}\right)+\alpha_{2} \dot{\mu} p_{2} q_{2}+\alpha_{2} \mu\left(q_{2} \dot{p}_{2}+p_{2} \dot{p}_{2} q_{2}^{\prime}\right) \\
-\left(\alpha_{1} c_{1} q_{1}^{\prime} \dot{p}_{1}+\alpha_{2} c_{2}\left(\dot{\mu} q_{2}+\mu q_{2}^{\prime} \dot{p}_{2}\right)\right)-C^{\prime}(\mu) \dot{\mu}=0 .
\end{gathered}
$$

The second-order condition for truth-telling is:

$$
-\alpha_{2}\left(\dot{\mu} q_{2}+\mu q_{2}^{\prime} \dot{p}_{2}\right) \geq 0
$$

Since $q_{2}^{\prime}<0$, a sufficient second-order condition is $\dot{\mu}<0$ and $\dot{p}_{2}>0$. So, the strategy is to solve the problem in the absence of the second-order condition and check ex post that the sufficient condition $\dot{\mu}<0$ and $\dot{p}_{2}>0$ is satisfied (see Guesnerie and Laffont (1984)).

We can easily represent the impact of asymmetric information on Figure 1 by looking at equations (18) (19). We obtain Figure 2. 


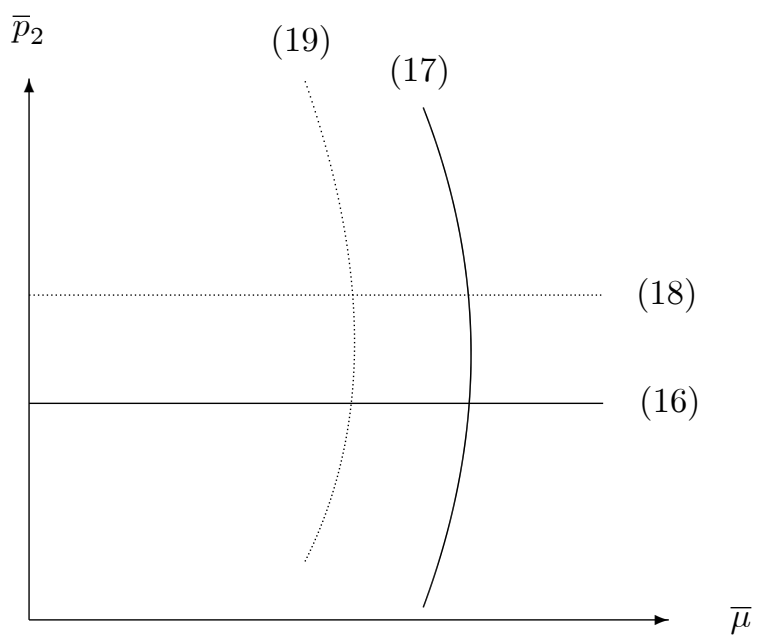

Figure 2

Therefore $\bar{p}_{2}^{S B}>\bar{p}_{2}^{*}>\underline{p}_{2}^{*}=\underline{p}_{2}^{S B}$ and $\bar{\mu}^{S B}<\bar{\mu}^{*}<\underline{\mu}^{*}=\underline{\mu}^{S B}$. So the second-order condition of incentive compatibility is satisfied.

\section{Proof of Proposition 3:}

The first-order conditions with respect to $\bar{q}$ and $\bar{\mu}$ are:

$$
\begin{aligned}
\left(\alpha_{1}+\alpha_{2} \bar{\mu}\right)\left(S^{\prime}(\bar{q})+\lambda \bar{p}^{\prime} \bar{q}+\lambda \bar{p}\right)-(1+\lambda)\left(\alpha_{1} c_{1}+\alpha_{2} \bar{c}_{2} \bar{\mu}\right) & =0 \\
\alpha_{2}(S(\bar{q})+\lambda \bar{p} \bar{q})-(1+\lambda)\left(\alpha_{2} \bar{c}_{2} \bar{q}+C^{\prime}(\bar{\mu})\right) & =0 .
\end{aligned}
$$

From second-order conditions, the objective function is concave if the following conditions are satisfied:

$$
\begin{gathered}
\left(\alpha_{1}+\alpha_{2} \bar{\mu}\right)\left(S^{\prime \prime}(\bar{q})+\lambda \bar{p}^{\prime \prime} \bar{q}+2 \lambda \bar{p}^{\prime}\right)<0 \\
-C^{\prime \prime}(\bar{\mu})\left(\alpha_{1}+\alpha_{2} \bar{\mu}\right)\left(S^{\prime \prime}(\bar{q})+\lambda \bar{p}^{\prime \prime} \bar{q}+2 \lambda \bar{p}\right)>(1+\lambda)\left[\frac{\alpha_{1} \alpha_{2}\left(c_{1}-c_{2}\right)}{\alpha_{1}+\alpha_{2} \bar{\mu}}\right]^{2} .
\end{gathered}
$$

So a sufficient condition for the concavity of the objective function is $\left|S^{\prime \prime}\right|$ large enough.

Since $\frac{\alpha_{1} c_{1}+\alpha_{2} c_{2} \mu}{\alpha_{1}+\alpha_{2} \mu}<c_{2}$, we have $\bar{p}^{* *}<\bar{p}_{2}^{*}$ and $\underline{p}^{* *}<\underline{p}_{2}^{*}$. Moreover, since $\bar{p}_{2}^{*}$ maximizes $\frac{S\left(\bar{q}_{2}\right)+\lambda \bar{p}_{2} \bar{q}_{2}}{1+\lambda}-\bar{c}_{2} \bar{q}_{2}$ and $\frac{S^{\prime}\left(\bar{q}^{* *}\right)+\lambda \bar{p}^{* * \prime} \bar{q}^{* *}+\lambda \bar{p}^{* *}}{1+\lambda}-\bar{c}_{2}<0$ by substituting the first-order conditions, one obtains that $\bar{\mu}^{* *}<\bar{\mu}^{*}$. Similarly, $\underline{\mu}^{* *}<\underline{\mu}^{*}$. 


\section{Proof of Proposition 4:}

The first-order conditions are:

$$
\begin{gathered}
-\nu \lambda \alpha_{1} \Delta c_{2} \bar{\mu}+(1-\nu)\left[\left(\alpha_{1}+\alpha_{2} \bar{\mu}\right)\left(S^{\prime}(\bar{q})+\lambda \bar{p}^{\prime} \bar{q}+\lambda \bar{p}\right)-(1+\lambda)\left(\alpha_{1} c_{1}+\alpha_{2} \bar{c}_{2} \bar{\mu}\right)\right]=0 \\
-\nu \lambda \alpha_{1} \Delta c_{2} \bar{q}+(1-\nu)\left[\alpha_{2}(S(\bar{q})+\lambda \overline{p q})-(1+\lambda)\left(\alpha_{2} \bar{c}_{2} \bar{q}+C^{\prime}(\bar{\mu})\right)\right]=0 .
\end{gathered}
$$

One can show that a sufficient condition for the concavity of the objective function is $\left|S^{\prime \prime}\right|$ large enough.

Denote $U\left(c_{2}, \widetilde{c}_{2}\right) \equiv t\left(\widetilde{c}_{2}\right)+\left(\alpha_{1}+\alpha_{2} \mu\left(\widetilde{c}_{2}\right)\right) p\left(q\left(\widetilde{c}_{2}\right)\right) q\left(\widetilde{c}_{2}\right)-\left(\alpha_{1} c_{1}+\alpha_{2} c_{2} \mu\left(\widetilde{c}_{2}\right)\right) q\left(\widetilde{c}_{2}\right)-$ $C\left(\mu\left(\widetilde{c}_{2}\right)\right)$ as the firm's utility when $\widetilde{c}_{2}$ is its report about $c_{2}$. The first-order condition for truth-telling is (for a continuous variable $c_{2}$ ):

$\dot{t}+\alpha_{2} \dot{\mu} p(q) q+\left(\alpha_{1}+\alpha_{2} \mu\right)\left(p^{\prime} \dot{q} q+p \dot{q}\right)-\alpha_{2} c_{2} \dot{\mu} q-\left(\alpha_{1} c_{1}+\alpha_{2} c_{2} \mu\right) \dot{q}-C^{\prime}(\mu) \dot{\mu}=0$.

The second-order condition is:

$$
-\alpha_{2} \dot{\mu} q-\alpha_{2} \mu \dot{q} \geq 0 .
$$

A sufficient condition for the second-order condition to be satisfied is $\bar{p}^{U S B}>$ $\underline{p}^{U S B}$ and $\bar{\mu}^{U S B}<\underline{\mu}^{U S B}$, which implies:

$$
\begin{gathered}
\frac{\alpha_{1} c_{1}+\alpha_{2} \bar{c}_{2} \bar{\mu}^{U S B}}{\alpha_{1}+\alpha_{2} \bar{\mu}^{U S B}}+\frac{\lambda}{1+\lambda} \frac{\nu}{1-\nu} \frac{\alpha_{2} \Delta c_{2} \bar{\mu}^{U S B}}{\alpha_{1}+\alpha_{2} \bar{\mu}^{U S B}}>\frac{\alpha_{1} c_{1}+\alpha_{2} \underline{c}_{2} \underline{\mu}^{* *}}{\alpha_{1}+\alpha_{2} \underline{\mu}^{* *}} \\
\frac{S\left(\bar{q}^{U S B}\right)+\lambda \bar{p}^{U S B} \bar{q}^{U S B}}{1+\lambda}-\bar{q}^{U S B} \bar{c}_{2}-\frac{\lambda}{1+\lambda} \frac{\nu}{1-\nu} \Delta c_{2} \bar{q}^{U S B}<\frac{S\left(\underline{q}^{* *}\right)+\lambda \underline{p}^{* *} \underline{q}^{* *}}{1+\lambda}-\underline{q}^{* *} \underline{c}_{2} .
\end{gathered}
$$

Thus a sufficient condition for the second-order condition of truth telling to be satisfied is $\Delta c_{2}$ large enough. However, if $\Delta c_{2}$ small enough, we have $\bar{p}^{U S B}<$ $\underline{p}^{U S B}$ and $\bar{\mu}^{U S B}>\underline{\mu}^{U S B}$. Then bunching occurs so that $\bar{p}^{U S B}=\underline{p}^{U S B}=p^{U S B}$ and $\bar{\mu}^{U S B}=\underline{\mu}^{U S B}=\mu^{U S B}$.

Let us now compute the condition that ensures $\bar{p}^{U S B}>\bar{p}^{* *}$ and $\bar{\mu}^{U S B}<\bar{\mu}^{* *}$.

From the first-order conditions we have:

$$
\frac{\alpha_{1} c_{1}+\alpha_{2} \bar{c}_{2} \bar{\mu}^{U S B}}{\alpha_{1}+\alpha_{2} \bar{\mu}^{U S B}}+\frac{\lambda}{1+\lambda} \frac{\nu}{1-\nu} \frac{\alpha_{2} \Delta c_{2} \bar{\mu}^{U S B}}{\alpha_{1}+\alpha_{2} \bar{\mu}^{U S B}}>\frac{\alpha_{1} c_{1}+\alpha_{2} \bar{c}_{2} \bar{\mu}^{* *}}{\alpha_{1}+\alpha_{2} \bar{\mu}^{* *}}
$$


or $\bar{p}^{U S B}>\bar{p}^{* *}$ if $\Delta c_{2}$ is large enough. Similarly, $\bar{\mu}^{U S B}<\bar{\mu}^{* *}$ if $\Delta c_{2}$ is large enough. Finally, since $\frac{\alpha_{1} c_{1}+\alpha_{2} \bar{c}_{2} \bar{\mu}^{U S B}}{\alpha_{1}+\alpha_{2} \bar{\mu}^{U S B}}+\frac{\lambda}{1+\lambda} \frac{\nu}{1-\nu} \frac{\alpha_{2} \Delta c_{2} \bar{\mu}^{U S B}}{\alpha_{1}+\alpha_{2} \bar{\mu}^{U S B}}$ is always smaller than $\bar{c}_{2}+\frac{\lambda}{1+\lambda} \frac{\nu}{1-\nu} \Delta c_{2}$, we have $\bar{p}^{U S B}<\bar{p}_{2}^{S B}$. Moreover, since $\bar{p}_{2}^{S B}$ optimizes $\frac{S\left(\bar{q}_{2}\right)+\lambda \bar{p}_{2} \bar{q}_{2}}{1+\lambda}-\bar{q}_{2} \bar{c}_{2}-\frac{\lambda}{1+\lambda} \frac{\nu}{1-\nu} \Delta c_{2} \bar{q}_{2}$, we have $\bar{\mu}^{U S B}<\bar{\mu}^{S B}$.

\section{Proof of Proposition 6:}

Define $\bar{q}^{u c}=q\left(c_{2}=\bar{c}_{2}, \sigma=\bar{c}_{2}\right), \underline{q}^{u c}=q\left(c_{2}=\underline{c}_{2}, \sigma=\underline{c}_{2}\right), \bar{q}^{u c \phi}=q\left(c_{2}=\right.$ $\left.\bar{c}_{2}, \sigma=\phi\right)$, and $\underline{q}^{u c \phi}=q\left(c_{2}=\underline{c}_{2}, \sigma=\phi\right)$. Other notation such as $\underline{\mu}^{u c}, \underline{\mu}^{u c \phi}, \bar{\mu}^{u c}$, and $\bar{\mu}^{u c \phi}$ is similarly defined.

If $c_{2}=\underline{c}_{2}$ and $\sigma=\underline{c}_{2}$, the good type firm will get an information rent of $\alpha_{2} \Delta c_{2} \bar{\mu}^{c \phi} \bar{q}^{c \phi}$ if the regulator hides the information. Thus the government has to satisfy the following collusion-proofness constraint to induce truth-telling by the regulator:

$$
\underline{s}^{u f} \geqslant k_{1} \alpha_{2} \Delta c_{2} \bar{\mu}^{u c \phi} \bar{q}^{u c \phi}
$$

As with discriminatory pricing, rural consumers do not have any stake in collusion because neither the price nor the size of the network is distorted if $c_{2}=$ $\underline{c}_{2}$. Since $c_{1}$ is common knowledge, urban consumers cannot collude with the regulator, even though they are adversely affected by uniform pricing. Similarly, taxpayers will pay the same amount of taxes regardless of the regulator's report.

Suppose the state of nature is $c_{2}=\bar{c}_{2}$ and $\sigma=\bar{c}_{2}$. The firm does not have any stake in collusion any more because it does not get any information rent when $c_{2}=\bar{c}_{2}$. We know from Proposition 4 that when asymmetric information is large enough, the price for the rural area is higher and the network smaller if the regulator hides his information. Thus $\alpha_{2} \bar{\mu}^{u c}\left(S\left(\bar{q}^{u c}\right)-\bar{p}^{u c} \bar{q}^{u c}\right)>$ $\alpha_{2} \bar{\mu}^{u c \phi}\left(S\left(\bar{q}^{u c \phi}\right)-\bar{p}^{u c \phi} \bar{q}^{u c \phi}\right)$ - that is, rural consumers obtain less surplus if the regulator does not report truthfully, so they have no incentive to collude. Sim-

ilarly, collusion between urban consumers and the regulator is not an issue in this state of nature.

Note that taxpayers pay $T^{u c}=\left(\alpha_{1} c_{1}+\alpha_{2} \bar{c}_{2} \bar{\mu}^{u c}\right) \bar{q}^{u c}-\left(\alpha_{1}+\alpha_{2} \bar{\mu}^{u c}\right) \bar{p}^{u c} \bar{q}^{u c}+$ $C\left(\bar{\mu}^{u c}\right)$ if the signal is revealed and $T^{u c \phi}=\left(\alpha_{1} c_{1}+\alpha_{2} \bar{c}_{2} \bar{\mu}^{c \phi}\right) \bar{q}^{u c \phi}-\left(\alpha_{1}+\right.$ 
$\left.\alpha_{2} \bar{\mu}^{u c \phi}\right) \bar{p}^{u c \phi} \bar{q}^{u c \phi}+C\left(\bar{\mu}^{u c \phi}\right)$ if not. Since $\left(\alpha_{1} c_{1}+\alpha_{2} \bar{c}_{2} \mu\right) q-\left(\alpha_{1}+\alpha_{2} \mu\right) p q+C(\mu)>$ 0 if $C^{\prime}(\cdot)$ is large enough, the taxpayers save a tax payment of $T^{u c}-T^{u c \phi}>0$ if the regulator reports $r=\phi$. So they have incentives to collude with the regulator. Thus the government needs to satisfy the following collusion-proofness constraint:

$$
\begin{aligned}
\bar{s}^{u t} \geqslant & k_{2}\left[\left(\alpha_{1} c_{1}+\alpha_{2} \bar{c}_{2} \bar{\mu}^{u c}\right) \bar{q}^{u c}-\left(\alpha_{1}+\alpha_{2} \bar{\mu}^{u c}\right) \bar{p}^{u c} \bar{q}^{u c}+C\left(\bar{\mu}^{u c}\right)\right. \\
& \left.-\left(\alpha_{1} c_{1}+\alpha_{2} \bar{c}_{2} \bar{\mu}^{u c \phi}\right) \bar{q}^{u c \phi}+\left(\alpha_{1}+\alpha_{2} \bar{\mu}^{u c \phi}\right) \bar{p}^{u c \phi} \bar{q}^{u c \phi}-C\left(\bar{\mu}^{u c \phi}\right)\right]
\end{aligned}
$$

Manipulation of first-order conditions leads to the following result:

$$
\begin{gathered}
\underline{p}^{u c}=\underline{p}^{u c \phi}=\underline{p}^{* *}, \underline{\mu}^{u c}=\underline{\mu}^{u c \phi}=\underline{\mu}^{* *} \\
\frac{\bar{p}^{c \phi}-\frac{\alpha_{1} c_{1}+\alpha_{2} \bar{c}_{2} \bar{\mu}^{c \phi}}{\alpha_{1}+\alpha_{2} \bar{\mu}^{c \phi}}\left(1-\frac{\lambda}{1+\lambda} \frac{\xi}{1-\xi} k_{2}\right)}{\bar{p}^{c \phi}}= \\
\frac{\lambda}{1+\lambda} \frac{1}{\eta}+\frac{\lambda}{1+\lambda} \frac{\xi}{1-\xi} k_{2}\left(1-\frac{1}{\eta}\right)+\frac{\lambda}{1+\lambda} \frac{\nu}{1-\nu} \frac{1}{1-\xi} \frac{\alpha_{2} \Delta c_{2} \bar{\mu}^{u c \phi}}{\alpha_{1}+\alpha_{2} \bar{\mu}^{u c \phi}} \frac{1}{\bar{p}^{u c \phi}}\left[\xi k_{1}+(1-\xi)\right] \\
\frac{\bar{p}^{u c}-\frac{\alpha_{1} c_{1}+\alpha_{2} \bar{c}_{2} \bar{\mu}^{u c}}{\alpha_{1}+\alpha_{2} \overline{\bar{m}}^{u c}}\left(1-\frac{\lambda}{1+\lambda} k_{2}\right)}{\bar{p}^{u c}}=\frac{\lambda}{1+\lambda} \frac{1}{\eta}-\frac{\lambda}{1+\lambda} k_{2}\left(1-\frac{1}{\eta}\right) \\
C^{\prime}\left(\bar{\mu}^{u c \phi}\right)\left(1-\frac{\lambda}{1+\lambda} \frac{\xi}{1-\xi} k_{2}\right)=\alpha_{2}\left[\frac{S\left(\bar{q}^{u c \phi}\right)+\lambda \bar{p}^{u c \phi} \bar{q}^{u c \phi}}{1+\lambda}-\bar{c}_{2} \bar{q}^{c \phi}-\frac{\lambda}{1+\lambda} \frac{\nu}{1-\nu} \frac{\bar{q}^{u c \phi} \Delta c_{2}}{1-\xi}\left(k_{1} \xi+(1-\xi)\right)\right. \\
C^{\prime}\left(\bar{\mu}^{u c}\right)\left(1+\frac{\lambda}{1+\lambda} \frac{\xi}{1-\xi} k_{2} \bar{q}^{u c \phi}\left(\bar{p}^{u c \phi}-\bar{c}_{2}\right)\right] \\
=\alpha_{2}\left[\frac{S\left(\bar{q}^{u c}\right)+\lambda \bar{p}^{u c} \bar{q}^{u c}}{1+\lambda}-\bar{c}_{2} \bar{q}^{u c}+\frac{\lambda}{1+\lambda} k_{2} \bar{q}^{u c}\left(\bar{p}^{c}-\bar{c}_{2}\right)\right] .
\end{gathered}
$$

